CARPATHIAN J. MATH.

Volume 38 (2022), No. 2,

Pages 337 - 346

\title{
On the crossing number of the join of the wheel on six vertices with a path
}

\author{
ŠTEFAN BEREŽNÝ and MICHAL STAŠ
}

\begin{abstract}
The crossing number $\operatorname{cr}(G)$ of a graph $G$ is the minimum number of edge crossings over all drawings of $G$ in the plane. The main aim of the paper is to give the crossing number of join product $W_{5}+P_{n}$ for the wheel $W_{5}$ on six vertices, where $P_{n}$ is the path on $n$ vertices. Staš and Valiska conjectured that the crossing number of $W_{m}+P_{n}$ is equal to $Z(m+1) Z(n)+(Z(m)-1)\left\lfloor\frac{n}{2}\right\rfloor+n+1$, for all $m \geq 3, n \geq 2$, where Zarankiewicz's number is defined as $Z(n)=\left\lfloor\frac{n}{2}\right\rfloor\left\lfloor\frac{n-1}{2}\right\rfloor$ for $n \geq 1$. Recently, this conjecture was proved for $W_{3}+P_{n}$ by Klešč and Schrötter, and for $W_{4}+P_{n}$ by Staš and Valiska. We establish the validity of this conjecture for $W_{5}+P_{n}$. The conjecture also holds due to some isomorphisms for $W_{m}+P_{2}, W_{m}+P_{3}$ by Klešč, and for $W_{m}+P_{4}$ by Staš for all $m \geq 3$.
\end{abstract}

\section{INTRODUCTION}

The crossing number $\operatorname{cr}(G)$ of a simple graph $G$ with the vertex set $V(G)$ and the edge set $E(G)$ is the minimum possible number of edge crossings in a drawing of $G$ in the plane. (For the definition of a drawing see Klešč [10].) It is easy to see that a drawing with the minimum number of crossings (an optimal drawing) is always a good drawing, meaning that no edge crosses itself, no two edges cross more than once, and no two edges incident with the same vertex cross. The join product of two graphs $G_{i}$ and $G_{j}$, denoted by $G_{i}+G_{j}$, is obtained from vertex-disjoint copies of $G_{i}$ and $G_{j}$ by adding all edges between $V\left(G_{i}\right)$ and $V\left(G_{j}\right)$. For $\left|V\left(G_{i}\right)\right|=m$ and $\left|V\left(G_{j}\right)\right|=n$, the edge set of $G_{i}+G_{j}$ is the union of disjoint edge sets of the graphs $G_{i}, G_{j}$, and the complete bipartite graph $K_{m, n}$.

Let $D$ be a good drawing of the graph $G$. We denote the number of crossings in $D$ by $\operatorname{cr}_{D}(G)$. Let $G_{i}$ and $G_{j}$ be edge-disjoint subgraphs of $G$. We denote the number of crossings between edges of $G_{i}$ and edges of $G_{j}$ by $\operatorname{cr}_{D}\left(G_{i}, G_{j}\right)$, and the number of crossings among edges of $G_{i}$ in $D$ by $\operatorname{cr}_{D}\left(G_{i}\right)$. For any three mutually edge-disjoint subgraphs $G_{i}$, $G_{j}$, and $G_{k}$ of $G$ by [10], the following equations hold:

$$
\begin{gathered}
\operatorname{cr}_{D}\left(G_{i} \cup G_{j}\right)=\operatorname{cr}_{D}\left(G_{i}\right)+\operatorname{cr}_{D}\left(G_{j}\right)+\operatorname{cr}_{D}\left(G_{i}, G_{j}\right), \\
\operatorname{cr}_{D}\left(G_{i} \cup G_{j}, G_{k}\right)=\operatorname{cr}_{D}\left(G_{i}, G_{k}\right)+\operatorname{cr}_{D}\left(G_{j}, G_{k}\right) .
\end{gathered}
$$

The investigation of the crossing number of graphs is a classical and very difficult problem. Garey and Johnson [7] proved that this problem is NP-complete. The exact values of the crossing numbers are known for some families of graphs, see Clancy et al. [4]. The purpose of this paper is to extend the known results concerning this topic. Some parts of proofs will be based on Kleitman's result [9] on the crossing numbers for some complete bipartite graphs. He showed that

$$
\operatorname{cr}\left(K_{m, n}\right)=\left\lfloor\frac{m}{2}\right\rfloor\left\lfloor\frac{m-1}{2}\right\rfloor\left\lfloor\frac{n}{2}\right\rfloor\left\lfloor\frac{n-1}{2}\right\rfloor, \quad \text { if } \quad m \leq 6 .
$$

Received: 08.03.2021. In revised form: 19.07.2021. Accepted: 26.07.2021

2010 Mathematics Subject Classification. 05C10, 05C38.

Key words and phrases. Graph, drawing, crossing number, join product, wheel, path.

Corresponding author: Michal Staš; michal.stas@tuke.sk 
Using Kleitman's result, the crossing numbers for the join product of paths with all graphs of order four were studied by Klešč [11] and Klešč and Schrötter [17]. The exact values for the crossing numbers of $G+P_{n}$ for some graphs $G$ on five vertices are determined in $[16,18,22,23,24,25]$. The crossing numbers of the join product $G+P_{n}$ are known only for a few graphs $G$ of order six, and so the purpose of this article is to extend the known results concerning this topic to new connected graphs, see $[2,5,6,10,13,20]$. Minimal number of crossings in the Cartesian product and in the strong product of paths have been studied by Klešč et al. [14] and [15].

Staš and Valiska [25] observed that the optimal drawing for $W_{4}+P_{n}$ can be generalized to drawings of $W_{m}+P_{n}$, which lead them to conjecture that the crossing number of $W_{m}+$ $P_{n}$ equals $Z(m+1) Z(n)+(Z(m)-1)\left\lfloor\frac{n}{2}\right\rfloor+n+1$ for all $m \geq 3, n \geq 2$, where $Z(n)=$ $\left\lfloor\frac{n}{2}\right\rfloor\left\lfloor\frac{n-1}{2}\right\rfloor$ is Zarankiewicz's number. Recently, this conjecture was proved for the crossing numbers of join products $W_{3}+P_{n}$ and $W_{4}+P_{n}$ by Klešč and Schrötter [17] and by Staš and Valiska [25], respectively. Results by Klešč [11] and [12] establish the conjecture for $W_{m}+P_{2}$ and $W_{m}+P_{3}$, and by Staš [23] for $W_{m}+P_{4}$.

The main purpose of the current paper is to show that the conjecture is true for $W_{5}+P_{n}$, for all $n \geq 2$.

Theorem 1.1. $\operatorname{cr}\left(W_{5}+P_{n}\right)=6\left\lfloor\frac{n}{2}\right\rfloor\left\lfloor\frac{n-1}{2}\right\rfloor+n+3\left\lfloor\frac{n}{2}\right\rfloor+1$ for $n \geq 2$.

Note that the result of Theorem 3.3 has already been claimed by Su and Huang [21] just as a conjecture without any proof. Clancy et al. [4] also placed an asterisk on a number of the results in their survey to essentially indicate that the mentioned results appeared in journals which do not have a sufficiently rigorous peer-review process. In the proofs of the paper, we will often use the term "region" also in nonplanar drawings. In this case, crossings are considered to be vertices of the "map".

\section{Cyclic Permutations}

This article follows definitions and notation for the crossing numbers from Klešč [11]. Let $W_{5}$ be the wheel on six vertices. We first consider the join product of $W_{5}$ with the discrete graph on $n$ vertices denoted by $D_{n}$. The graph $W_{5}+D_{n}$ consists of just one copy of the graph $W_{5}$ and of $n$ vertices $t_{1}, t_{2}, \ldots, t_{n}$, where each vertex $t_{i}, i=1,2, \ldots, n$, is adjacent to every vertex of $W_{5}$. Let $T^{i}, 1 \leq i \leq n$, denote the subgraph induced by the six edges incident with the vertex $t_{i}$. This means that the graph $T^{1} \cup \cdots \cup T^{n}$ is isomorphic to the complete bipartite graph $K_{6, n}$ and

$$
W_{5}+D_{n}=W_{5} \cup K_{6, n}=W_{5} \cup\left(\bigcup_{i=1}^{n} T^{i}\right) .
$$

The graph $W_{5}+P_{n}$ contains $W_{5}+D_{n}$ as a subgraph. For the subgraphs of the graph $W_{5}+P_{n}$ which are also subgraphs of the graph $W_{5}+n K_{1}$ we use the same notation as above. Let $P_{n}^{*}$ denote the path induced by $n$ vertices of $W_{5}+P_{n}$ not belonging to the subgraph $W_{5}$. Hence, $P_{n}^{*}$ consists of the vertices $t_{1}, t_{2}, \ldots, t_{n}$ and of the edges $\left\{t_{i}, t_{i+1}\right\}$ for $i=1,2, \ldots, n-1$. One can easily see that

$$
W_{5}+P_{n}=W_{5} \cup K_{6, n} \cup P_{n}^{*}=W_{5} \cup\left(\bigcup_{i=1}^{n} T^{i}\right) \cup P_{n}^{*} .
$$

Let $D$ be a good drawing of the graph $W_{5}+D_{n}$. The rotation $\operatorname{rot}_{D}\left(t_{i}\right)$ of a vertex $t_{i}$ in the drawing $D$ is the cyclic permutation that records the (cyclic) counterclockwise order in which the edges leave $t_{i}$, as defined by Hernández-Vélez et al. [8] or Woodall [26]. We 
use the notation (123456) if the counter-clockwise order the edges incident with the vertex $t_{i}$ is $t_{i} v_{1}, t_{i} v_{2}, t_{i} v_{3}, t_{i} v_{4}, t_{i} v_{5}$, and $t_{i} v_{6}$. Recall that a rotation is a cyclic permutation; that is, (123456), (234561), (345612), (456123), (561234), and (612345) denote the same rotation. We separate all subgraphs $T^{i}, i=1,2, \ldots, n$, of the graph $W_{5}+D_{n}$ into four mutuallydisjoint families of subgraphs depending on how many times the considered $T^{i}$ crosses the edges of $W_{5}$ in $D$. Let $R_{D}=\left\{T^{i}: \mathrm{cr}_{D}\left(W_{5}, T^{i}\right)=0\right\}, S_{D}=\left\{T^{i}: \operatorname{cr}_{D}\left(W_{5}, T^{i}\right)=1\right\}$, and $T_{D}=\left\{T^{i}: \operatorname{cr}_{D}\left(W_{5}, T^{i}\right)=2\right\}$. Every other subgraph $T^{i}$ crosses the edges of $W_{5}$ at least three times in $D$. For $T^{i} \in R_{D} \cup S_{D} \cup T_{D}$, let $F^{i}$ denote the subgraph $W_{5} \cup T^{i}$, $i \in\{1,2, \ldots, n\}$, of $W_{5}+D_{n}$ and let $D\left(F^{i}\right)$ be its good subdrawing induced by $D$. Clearly, the four families we mentioned are the same in the drawing $D$ of $W_{5}+P_{n}$ and in the subdrawing $D^{\prime}$ of $W_{5}+D_{n}$ induced by $D$ without the edges of $P_{n}^{*}$.

\section{Possible Drawings of $W_{5}$ And the Crossing Number of $W_{5}+P_{n}$}

Since the graph $W_{5}$ consists of one dominating vertex of degree 5 and of five vertices of degree 3 which form the subgraph isomorphic to the cycle $C_{5}$ (for brevity, we will write $C_{5}^{\star}$ ), we only need to consider possibilities of crossings between subdrawings of $C_{5}^{\star}$ and the five edges incident with the dominating vertex which form the subgraph isomorphic to the star $S_{5}$ on six vertices (also for brevity, we will write $S_{5}^{\star}$ ). In the rest of the paper, let $V\left(W_{5}\right)=\left\{v_{1}, v_{2}, \ldots, v_{6}\right\}$, and let $v_{6}$ be the vertex notation of the dominating vertex of degree 5 in all considered good subdrawings of the graph $W_{5}$.

Let us first note that if $D$ is a good drawing of $W_{5}+P_{n}$ with the empty set $R_{D} \cup S_{D}$, then $t=\left|T_{D}\right|<\left\lceil\frac{n}{2}\right\rceil$ implies at least $6\left\lfloor\frac{n}{2}\right\rfloor\left\lfloor\frac{n-1}{2}\right\rfloor+n+3\left\lfloor\frac{n}{2}\right\rfloor+1$ crossings in $D$ provided by

$$
\begin{gathered}
\operatorname{cr}_{D}\left(W_{5}+P_{n}\right) \geq \operatorname{cr}_{D}\left(K_{6, n}\right)+\operatorname{cr}_{D}\left(W_{5}, K_{6, n}\right) \geq 6\left\lfloor\frac{n}{2}\right\rfloor\left\lfloor\frac{n-1}{2}\right\rfloor+2 t+3(n-t)= \\
6\left\lfloor\frac{n}{2}\right\rfloor\left\lfloor\frac{n-1}{2}\right\rfloor+3 n-t \geq 6\left\lfloor\frac{n}{2}\right\rfloor\left\lfloor\frac{n-1}{2}\right\rfloor+3 n+1-\left\lceil\frac{n}{2}\right\rfloor \geq 6\left\lfloor\frac{n}{2}\right\rfloor\left\lfloor\frac{n-1}{2}\right\rfloor+n+3\left\lfloor\frac{n}{2}\right\rfloor+1 .
\end{gathered}
$$

Lemma 3.1. Let $G \in\left\{D_{n} \mid n \geq 1\right\} \cup\left\{P_{n} \mid n \geq 2\right\}$. In any optimal drawing of the join product $W_{5}+G$, the edges of $C_{5}^{\star}$ do not cross each other. Moreover, the subdrawing of $W_{5}$ induced by $D$, in which no two edges of $C_{5}^{\star}$ are crossed by any edge of $S_{5}^{\star}$ and with a possibility of obtaining a subgraph $T^{i}$ whose edges cross the edges of $W_{5}$ at most twice, is isomorphic to one of the eight drawings depicted in Fig. 1.

Proof. The proof for $W_{5}+D_{n}$ has already been presented by Berežný and Staš [3]. In the case of $W_{5}+P_{n}$, the proof uses the same idea, that is, we can always redraw a crossing of two edges of $C_{5}^{\star}$ to get a new drawing of $C_{5}^{\star}$ (with vertices in a different order) with less number of edge crossings.

Let $D$ be a good drawing of $W_{5}+D_{n}$ with no crossing among edges of $C_{5}^{\star}$, and let there be a possibility of obtaining a subgraph $T^{i}$ by which the edges of $W_{5}$ are crossed at most twice. Without lost of generality, let us denote by $v_{1} v_{2} v_{3} v_{4} v_{5} v_{1}$ the vertex notation of the cycle $C_{5}^{\star}$. Because any edge of $S_{5}^{\star}$ can cross at most one edge of $C_{5}^{\star}$, only three main cases need to be considered:

If no edge of the cycle $C_{5}^{\star}$ is crossed by any edge of $S_{5}^{\star}$, we obtain the planar subdrawing of $W_{5}$ shown in Fig. 1(a). For any $i=1, \ldots, 5$, if the edge $v_{i} v_{6}$ crosses some edge of $C_{5}^{\star}$ and all three regions of $D\left(C_{5}^{\star} \cup v_{i} v_{6}\right)$ contain at least three vertices of the graph $W_{5}$ on its boundary, then we obtain a subdrawing of $W_{5}$ isomorphic to the drawing shown in Fig. 1(c). Now, without lost of generality, let the edge $v_{3} v_{4}$ be crossed by the edge $v_{2} v_{6}$. The edge $v_{3} v_{6}$ must be without any crossing in $D\left(W_{5}\right)$, and the edge $v_{1} v_{6}$ either crosses one of the edges $v_{4} v_{5}, v_{3} v_{4}$ or does not cross the edges of $C_{5}^{\star}$. If $v_{1} v_{6}$ crosses $v_{4} v_{5}$, we obtain the subdrawing of $W_{5}$ shown in Fig. 1(e). If $v_{1} v_{6}$ crosses $v_{3} v_{4}$ and also $v_{4} v_{6}$ crosses $v_{1} v_{5}$, 
we obtain the subdrawing of $W_{5}$ shown in Fig. $1(\mathrm{~g})$. If $v_{1} v_{6}$ crosses $v_{3} v_{4}$ and also $v_{4} v_{6}$ is without any crossing in $D\left(W_{5}\right)$, then the edge $v_{5} v_{6}$ either does not cross any edge of $C_{5}^{\star}$ or cross the edge $v_{3} v_{4}$ and we obtain the subdrawings of $W_{5}$ shown in Fig. 1(f) and (h), respectively. Finally, if the edge $v_{1} v_{6}$ does not cross any edge of the cycle $C_{5}^{\star}$, then the edge $v_{5} v_{6}$ either also does not cross any edge of $C_{5}^{\star}$ or cross the edge $v_{3} v_{4}$ and we obtain the last two possible subdrawings of $W_{5}$ shown in Fig. 1(b) and (d), respectively.

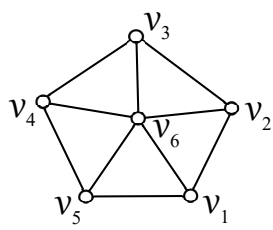

(a)

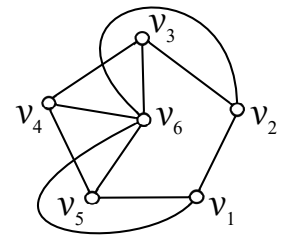

(e)

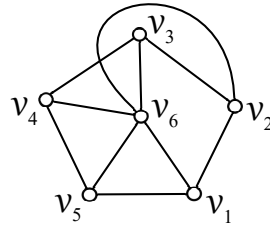

(b)

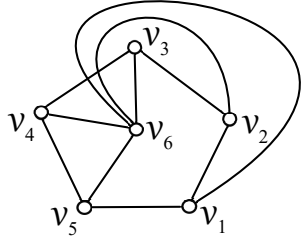

(f)

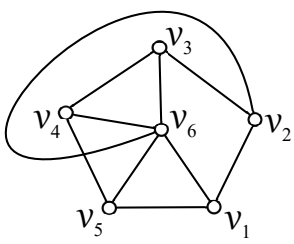

(c)

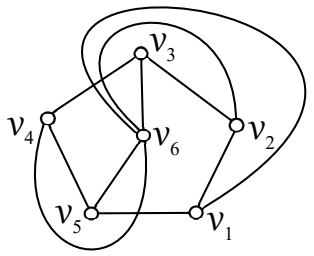

(g)

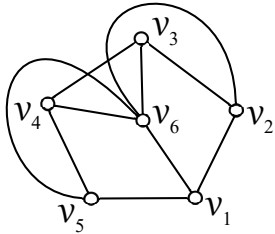

(d)

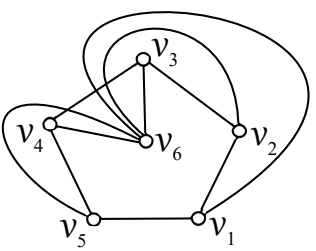

(h)

FIGURE 1. Eight possible non isomorphic drawings of the graph $W_{5}$ with no crossing among edges of $C_{5}^{\star}$, where no two edges of $C_{5}^{\star}$ are crossed by any edge of $S_{5}^{\star}$, and also with a possibility of obtaining a subgraph $T^{i} \in R_{D} \cup S_{D} \cup T_{D}$.

Lemma 3.2. Let $D$ be a good drawing of $W_{5}+P_{n}, n \geq 2$. If the edges of $C_{5}^{\star}$ are crossed at least $\left\lceil\frac{n}{2}\right\rceil+1$ times, then there are at least $6\left\lfloor\frac{n}{2}\right\rfloor\left\lfloor\frac{n-1}{2}\right\rfloor+n+3\left\lfloor\frac{n}{2}\right\rfloor+1$ crossings in $D$.

Proof. As the wheel $W_{5}$ consists of two edge-disjoint subgraphs $C_{5}^{\star}$ and $S_{5}^{\star}$, let us consider that $\operatorname{cr}_{D}\left(C_{5}^{\star}\right)+\operatorname{cr}_{D}\left(C_{5}^{\star}, S_{5}^{\star}+P_{n}\right) \geq\left\lceil\frac{n}{2}\right\rceil+1$ is fulfilling in the good drawing $D$ of $W_{5}+P_{n}$. The star $S_{5}^{\star}$ is isomorphic to the complete bipartite graph $K_{1,5}$ and the exact value for the crossing number of $K_{1,5}+P_{n}$ as a direct corollary of the crossing number of $K_{1,5}+D_{n}$ is given by Mei and Huang [19], that is, $\operatorname{cr}\left(K_{1,5, n}\right)=\operatorname{cr}\left(K_{1,5}+P_{n}\right)=6\left\lfloor\frac{n}{2}\right\rfloor\left\lfloor\frac{n-1}{2}\right\rfloor+4\left\lfloor\frac{n}{2}\right\rfloor$. This enforces that the edges of $S_{5}^{\star}+P_{n}$ must be crossed at least $6\left\lfloor\frac{n}{2}\right\rfloor\left\lfloor\frac{n-1}{2}\right\rfloor+4\left\lfloor\frac{n}{2}\right\rfloor$ times in $D$. Consequently, we have

$$
\begin{gathered}
\operatorname{cr}_{D}\left(W_{5}+P_{n}\right)=\operatorname{cr}_{D}\left(S_{5}^{\star}+P_{n}\right)+\operatorname{cr}_{D}\left(C_{5}^{\star}\right)+\operatorname{cr}_{D}\left(C_{5}^{\star}, S_{5}^{\star}+P_{n}\right) \\
\geq 6\left\lfloor\frac{n}{2}\right\rfloor\left\lfloor\frac{n-1}{2}\right\rfloor+4\left\lfloor\frac{n}{2}\right\rfloor+\left\lceil\frac{n}{2}\right\rfloor+1=6\left\lfloor\frac{n}{2}\right\rfloor\left\lfloor\frac{n-1}{2}\right\rfloor+n+3\left\lfloor\frac{n}{2}\right\rfloor+1 .
\end{gathered}
$$

Corollary 3.1. Let $D$ be a good drawing of $W_{5}+P_{n}, n \geq 2$, with no crossing among edges of $C_{5}^{\star}$, and let $\left|T_{D}\right| \geq\left\lceil\frac{n}{2}\right\rceil$. If any subgraph $T^{i} \in T_{D}$ crosses some edge of the cycle $C_{5}^{\star}$, then there are at least $6\left\lfloor\frac{n}{2}\right\rfloor\left\lfloor\frac{n-1}{2}\right\rfloor+n+3\left\lfloor\frac{n}{2}\right\rfloor+1$ crossings in $D$. 
Proof. In the planar drawing of $W_{5}$ (shown in Fig. 1(a)), there is no possibility to obtain a subdrawing of $W_{5} \cup T^{i}$ for a subgraph $T^{i} \in T_{D}$. All cases of good drawings of $W_{5}+P_{n}$ with at least one crossing among edges of the graph $W_{5}$ enforce $\operatorname{cr}_{D}\left(C_{5}^{\star}, S_{5}^{\star}\right) \geq 1$. So, if $\left|T_{D}\right| \geq\left\lceil\frac{n}{2}\right\rceil$ and any $T^{i} \in T_{D}$ crosses some edge of $C_{5}^{\star}$, then the edges of $C_{5}^{\star}$ are crossed at least $\left\lceil\frac{n}{2}\right\rceil+1$ times, and therefore by Lemma 3.2, there are at least $6\left\lfloor\frac{n}{2}\right\rfloor\left\lfloor\frac{n-1}{2}\right\rfloor+n+3\left\lfloor\frac{n}{2}\right\rfloor+1$ crossings in $D$.

Corollary 3.2. Let $D$ be a good drawing of $W_{5}+P_{n}, n \geq 2$, with no crossing among edges of $C_{5}^{*}$, and let all vertices $t_{i}$ of the path $P_{n}^{*}$ be placed in the same region of the considered good subdrawing of $W_{5}$. If at least two edges of $C_{5}^{\star}$ are crossed by the same edge of $S_{5}^{\star}$, then there are at least $6\left\lfloor\frac{n}{2}\right\rfloor\left\lfloor\frac{n-1}{2}\right\rfloor+n+3\left\lfloor\frac{n}{2}\right\rfloor+1$ crossings in $D$.

Proof. Let $D$ be a good drawing of $W_{5}+P_{n}$ with no crossing among edges of $C_{5}^{\star}$. If any subgraph $T^{i}$ crosses the edges of $W_{5}$ at least twice, then $\left|T_{D}\right| \geq\left\lceil\frac{n}{2}\right\rceil$ or there are at least $6\left\lfloor\frac{n}{2}\right\rfloor\left\lfloor\frac{n-1}{2}\right\rfloor+n+3\left\lfloor\frac{n}{2}\right\rfloor+1$ crossings in $D$.

Let us turn to the good drawing $D$ of the graph $W_{5}+P_{n}$ with the assumption that all vertices of $P_{n}^{*}$ are placed in the same region of the considered good subdrawing of $W_{5}$, and some edge of $S_{5}^{\star}$ crosses at least two different edges of $C_{5}^{\star}$. For this purpose, we suppose the drawing with the vertex notation of $W_{5}$ in such a way as shown in Fig. 2(a).

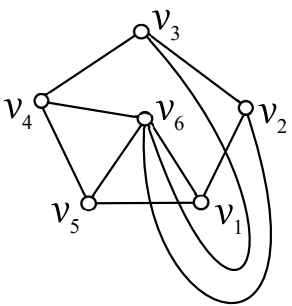

(a)

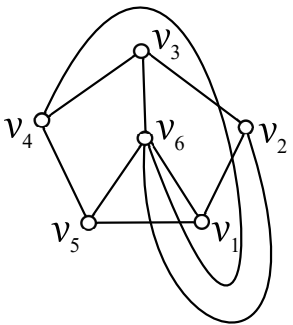

(b)

FIGURE 2. Two good drawings of the graph $W_{5}$ in which at least two edges of $C_{5}^{\star}$ are crossed by the same edge of $S_{5}^{\star}$.

Because no region is incident to at least five vertices in the subdrawing $D\left(W_{5}\right)$, there is no possibility to obtain a subdrawing of $W_{5} \cup T^{i}$ for a $T^{i} \in R_{D} \cup S_{D}$. As $r=0$ and $s=0$, we can assume that $\left|T_{D}\right| \geq\left\lceil\frac{n}{2}\right\rceil$, otherwise, we obtain the considered number of crossings in $D$. If all vertices of the path $P_{n}^{*}$ are placed in the outer region with four vertices $v_{2}, v_{3}$, $v_{4}$, and $v_{5}$ of the graph $W_{5}$ on its boundary, then the edges $t_{i} v_{6}$ cross some edge of the cycle $C_{5}^{\star}$, and therefore by Corollary 3.1, there are at least $6\left\lfloor\frac{n}{2}\right\rfloor\left\lfloor\frac{n-1}{2}\right\rfloor+n+3\left\lfloor\frac{n}{2}\right\rfloor+1$ crossings in $D$ again. Now, let all vertices of $P_{n}^{*}$ be placed in the inner region with four vertices $v_{1}, v_{3}$, $v_{4}$, and $v_{6}$ of $W_{5}$ on its boundary. The graph $W_{5}$ contains the cycle $v_{3} v_{4} v_{6} v_{3}$ as a subgraph by which the vertices $v_{2}, v_{5}$ and $t_{i}$ are separated in $D\left(W_{5}\right)$, that is, each subgraph $T^{i}$ crosses the edges of the 3-cycle $v_{3} v_{4} v_{6} v_{3}$ at least twice in $D\left(W_{5} \cup T^{i}\right)$. If the edges of $C_{5}^{\star}$ are crossed by all subgraphs $T^{i}$ at most $\left\lceil\frac{n}{2}\right\rceil-\operatorname{cr}_{D}\left(W_{5}\right)$ times due to Lemma 3.2, then there are at least $2 n-\left(\left\lceil\frac{n}{2}\right\rceil-3\right)+2$ crossings on the edges $v_{3} v_{6}$ and $v_{4} v_{6}$ in $D$. Let us denote by $H$ the subgraph of $W_{5}$ with the vertex set $V\left(W_{5}\right)$, and the edge set $E\left(W_{5}\right) \backslash\left\{v_{3} v_{6}, v_{4} v_{6}\right\}$. Since the exact value for the crossing number of the join product $H \backslash\left\{v_{1} v_{6}, v_{3} v_{4}\right\}+D_{n}$ is given in [1], i.e., $\operatorname{cr}\left(H \backslash\left\{v_{1} v_{6}, v_{3} v_{4}\right\}+D_{n}\right)=6\left\lfloor\frac{n}{2}\right\rfloor\left\lfloor\frac{n-1}{2}\right\rfloor+2\left\lfloor\frac{n}{2}\right\rfloor$, the edges of $H+P_{n}$ are crossed at least $6\left\lfloor\frac{n}{2}\right\rfloor\left\lfloor\frac{n-1}{2}\right\rfloor+2\left\lfloor\frac{n}{2}\right\rfloor$ times in $D$. Thus, the edges of $W_{5}+P_{n}$ are crossed at least $6\left\lfloor\frac{n}{2}\right\rfloor\left\lfloor\frac{n-1}{2}\right\rfloor+2\left\lfloor\frac{n}{2}\right\rfloor+2 n-\left(\left\lceil\frac{n}{2}\right\rceil-3\right)+2$ times in $D$. 
This result completes the proof for the considered subdrawing of $W_{5}$ in $D$ given in Fig. 2(a), and the proof proceeds in the similar way also for the remaining good subdrawings of $W_{5}$ in which one edge of $S_{5}^{\star}$ crosses at least two different edges of the cycle $C_{5}^{\star}$. In several cases (shown in Fig. 2(b)), it is sufficient to use only Corollary 3.1.

According to Lemma 3.1, the edges of the cycle $C_{5}^{\star}$ do not cross each other in any optimal drawing $D$ of the join product $W_{5}+P_{n}$. Using Corollary 3.2, we will consider only eight possible non isomorphic drawings of $W_{5}$ as shown in Fig. 1, in which there is no crossing among edges of $C_{5}^{\star}$, no two edges of $C_{5}^{\star}$ are crossed by any edge of $S_{5}^{\star}$, and there is a possibility of obtaining a subgraph $T^{i}$ by which the edges of $W_{5}$ are crossed at most twice. In the proof of Theorem 3.3, several parts are also based on the following Theorem 3.2.

Theorem 3.2 ([3], Theorem 3.1). $\operatorname{cr}\left(W_{5}+D_{n}\right)=6\left\lfloor\frac{n}{2}\right\rfloor\left\lfloor\frac{n-1}{2}\right\rfloor+n+3\left\lfloor\frac{n}{2}\right\rfloor$ for $n \geq 1$.

Even though we can compute the exact values of crossing numbers of two graphs $W_{5}+$ $P_{2}$ and $W_{5}+P_{3}$ using algorithm located on the website http://crossings.uos.de/, due to the simplicity of these proofs, we prove the following Lemma 3.3.

Lemma 3.3. $\operatorname{cr}\left(W_{5}+P_{2}\right)=6$ and $\operatorname{cr}\left(W_{5}+P_{3}\right)=13$.

Proof. The graphs $W_{5}+P_{2}$ and $W_{5}+P_{3}$ are isomorphic to the join product of the cycle $C_{5}$ with the cycle $C_{3}$ and with the graph $K_{4} \backslash e$ obtained by removing one edge from the complete graph $K_{4}$, respectively. In [11] and [12] were proved that $\operatorname{cr}\left(C_{5}+C_{3}\right)=6$ and $\operatorname{cr}\left(C_{5}+K_{4} \backslash e\right)=13$, respectively, and so $\operatorname{cr}\left(W_{5}+P_{2}\right)=6$ and $\operatorname{cr}\left(W_{5}+P_{3}\right)=13$.

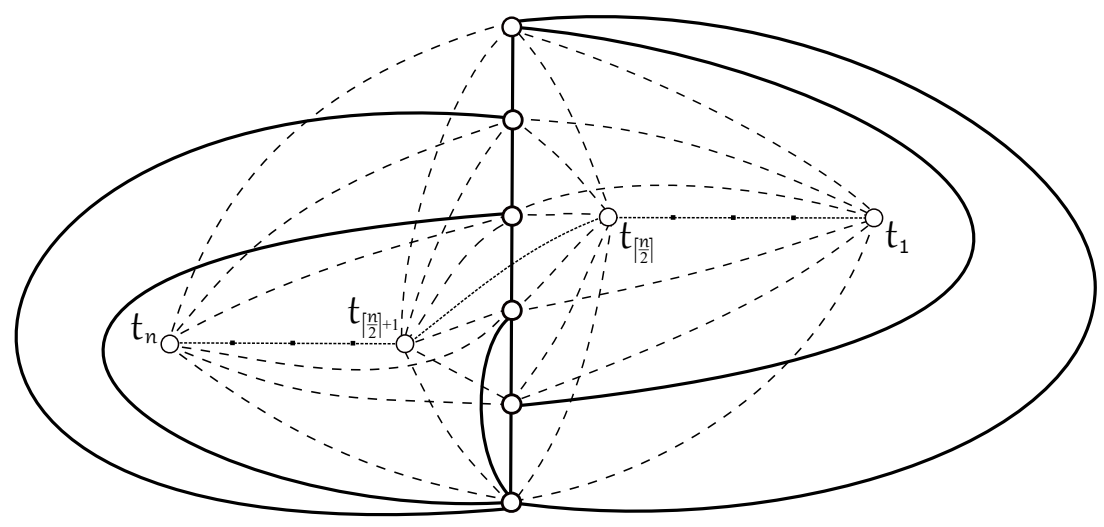

FIGURE 3. The good drawing of $W_{5}+P_{n}$ with $6\left\lfloor\frac{n}{2}\right\rfloor\left\lfloor\frac{n-1}{2}\right\rfloor+n+3\left\lfloor\frac{n}{2}\right\rfloor+1$ crossings.

Theorem 3.3. $\operatorname{cr}\left(W_{5}+P_{n}\right)=6\left\lfloor\frac{n}{2}\right\rfloor\left\lfloor\frac{n-1}{2}\right\rfloor+n+3\left\lfloor\frac{n}{2}\right\rfloor+1$ for $n \geq 2$.

Proof. In Fig. 3, the edges of $K_{6, n}$ cross each other $6\left\lfloor\frac{n}{2}\right\rfloor\left\lfloor\frac{n-1}{2}\right\rfloor$ times, each subgraph $T^{i}$, $i=1, \ldots,\left\lceil\frac{n}{2}\right\rceil$ on the right side crosses the edges of $C_{5}^{\star}$ exactly once and each subgraph $T^{i}, i=\left\lceil\frac{n}{2}\right\rceil+1, \ldots, n$ on the left side crosses the edges of $S_{5}^{\star}$ exactly four times. The path $P_{n}^{*}$ crosses $W_{5}$ once, and so $6\left\lfloor\frac{n}{2}\right\rfloor\left\lfloor\frac{n-1}{2}\right\rfloor+n+3\left\lfloor\frac{n}{2}\right\rfloor+1$ crossings appear among the edges of the graph $W_{5}+P_{n}$ in this drawing. Thus, $\operatorname{cr}\left(W_{5}+P_{n}\right) \leq 6\left\lfloor\frac{n}{2}\right\rfloor\left\lfloor\frac{n-1}{2}\right\rfloor+n+3\left\lfloor\frac{n}{2}\right\rfloor+1$. By Lemma 3.3, the result is true for $n=2$ and $n=3$. We prove the reverse inequality by induction on $n$. Suppose now that, for some $n \geq 4$, there is a drawing $D$ with

$$
\operatorname{cr}_{D}\left(W_{5}+P_{n}\right)<6\left\lfloor\frac{n}{2}\right\rfloor\left\lfloor\frac{n-1}{2}\right\rfloor+n+3\left\lfloor\frac{n}{2}\right\rfloor+1
$$


and that

$$
\operatorname{cr}\left(W_{5}+P_{m}\right)=6\left\lfloor\frac{m}{2}\right\rfloor\left\lfloor\frac{m-1}{2}\right\rfloor+m+3\left\lfloor\frac{m}{2}\right\rfloor+1 \text { for any integer } 2 \leq m<n .
$$

As the graph $W_{5}+D_{n}$ is a subgraph of the graph $W_{5}+P_{n}$, by Theorem 3.2 , the edges of $W_{5}+P_{n}$ are crossed exactly $6\left\lfloor\frac{n}{2}\right\rfloor\left\lfloor\frac{n-1}{2}\right\rfloor+n+3\left\lfloor\frac{n}{2}\right\rfloor$ times, and therefore, no edge of the path $P_{n}^{*}$ is crossed in $D$. This also enforces that all vertices $t_{i}$ of the path $P_{n}^{*}$ must be placed in the same region of the considered good subdrawing of $W_{5}$. Moreover, if $r=\left|R_{D}\right|, s=\left|S_{D}\right|$, and $t=\left|T_{D}\right|$, the assumption (3.3) together with the well-known fact $\operatorname{cr}\left(K_{6, n}\right)=6\left\lfloor\frac{n}{2}\right\rfloor\left\lfloor\frac{n-1}{2}\right\rfloor$ imply that, in $D$, if $r=0$ and $s=0$, then there are at least $\left\lceil\frac{n}{2}\right\rceil$ subgraphs $T^{i}$ by which the edges of $W_{5}$ are crossed exactly twice. More precisely:

$$
\operatorname{cr}_{D}\left(W_{5}\right)+\operatorname{cr}_{D}\left(W_{5}, K_{6, n}\right) \leq n+3\left\lfloor\frac{n}{2}\right\rfloor
$$

i.e.,

$$
\mathrm{cr}_{D}\left(W_{5}\right)+0 r+1 s+2 t+3(n-r-s-t) \leq n+3\left\lfloor\frac{n}{2}\right\rfloor .
$$

This forces that $3 r+2 s+t \geq\left\lceil\frac{n}{2}\right\rceil+\operatorname{cr}_{D}\left(W_{5}\right)$, and therefore, $t \geq\left\lceil\frac{n}{2}\right\rceil+\operatorname{cr}_{D}\left(W_{5}\right)$ if both sets $R_{D}$ and $S_{D}$ are empty. By Lemma 3.1, we can also suppose that there is no crossing among edges of $C_{5}^{\star}$ in all contemplated subdrawings of the graph $W_{5}$. Now, we will deal with the possibilities of obtaining a subgraph $T^{i} \in R_{D} \cup S_{D} \cup T_{D}$ in the drawing $D$ and we show that in all cases a contradiction with the assumption (3.3) is obtained. Recall that the drawing $D$ is also satisfying the restriction of placement all vertices $t_{i}$ in the same region of the considered subdrawing of $W_{5}$.

Case 1: $\operatorname{cr}_{D}\left(W_{5}\right)=0$. The drawing of $W_{5}$ is uniquely determined in such a way as shown in Fig. 1(a). It is obvious that the sets $R_{D}$ and $T_{D}$ are empty. As the set $S_{D}$ cannot be empty, all vertices $t_{i}$ must be placed in the region of $D\left(W_{5}\right)$ with five vertices of the graph $W_{5}$ on its boundary. Since each subgraph $T^{i}$ crosses some edge of $C_{5}^{\star}$ at least once, the edges of the cycle $C_{5}^{\star}$ are crossed at least $n$ times. Lemma 3.2 forces a contradiction with the assumption (3.3) in $D$.

Case 2: $\operatorname{cr}_{D}\left(W_{5}\right)=1$. At first, without loss of generality, we suppose the drawing with the vertex notation of $W_{5}$ in such a way as shown in Fig. 1(b). Since the sets $R_{D}$ and $S_{D}$ are empty, there are at least $\left\lceil\frac{n}{2}\right\rceil+1$ subgraphs $T^{i}$ whose edges cross the edges of $W_{5}$ exactly twice. It is easy to see that $\operatorname{cr}_{D}\left(C_{5}^{\star}, T^{i}\right) \geq 1$ holds for each such subgraph $T^{i} \in T_{D}$, and so Lemma 3.2 again contradicts the assumptions of $D$.

In addition, without loss of generality, we can choose the vertex notation of the graph $W_{5}$ in such a way as shown in Fig. 1(c). Clearly, also the sets $R_{D}$ and $S_{D}$ are empty, that is, $t \geq\left\lceil\frac{n}{2}\right\rceil+1$. Moreover, all vertices $t_{i}$ must be placed in the region of $D\left(W_{5}\right)$ with four vertices $v_{1}, v_{2}, v_{3}$, and $v_{6}$ of $W_{5}$ on its boundary. For a $T^{i} \in T_{D}$, there is only one subdrawing of $F^{i} \backslash\left\{v_{4}, v_{5}\right\}$ represented by the rotation (1236), which yields that there are four ways to obtain the subdrawing of $F^{i}$ depending on which two edges of $W_{5}$ are crossed by the edges $t_{i} v_{4}$ and $t_{i} v_{5}$. The edges of $C_{5}^{\star}$ are not crossed by any $T^{i} \in T_{D}$ only if $\operatorname{rot}_{D}\left(t_{i}\right)=(123465)$. It is not difficult to verify in six possible regions of $D\left(W_{5} \cup T^{i}\right)$ that $\operatorname{cr}_{D}\left(W_{5} \cup T^{i}, T^{k}\right) \geq 6$ is true for any subgraph $T^{k}$ with $k \neq i$. Thus, by fixing the subgraph $W_{5} \cup T^{i}$, we have

$$
\operatorname{cr}_{D}\left(W_{5}+P_{n}\right) \geq 6\left\lfloor\frac{n-1}{2}\right\rfloor\left\lfloor\frac{n-2}{2}\right\rfloor+6(n-1)+3 \geq 6\left\lfloor\frac{n}{2}\right\rfloor\left\lfloor\frac{n-1}{2}\right\rfloor+n+3\left\lfloor\frac{n}{2}\right\rfloor+1 .
$$

If there is no $T^{i} \in T_{D}$ with $\operatorname{rot}_{D}\left(t_{i}\right)=(123465)$, then each subgraph $T^{i} \in T_{D}$ crosses some edge of $C_{5}^{\star}$ at least once, and so Lemma 3.2 also confirms a contradiction with the assumption (3.3) in $D$. 
Case 3: $\operatorname{cr}_{D}\left(W_{5}\right)=2$. At first, without loss of generality, we can choose the vertex notation of the graph $W_{5}$ in such a way as shown in Fig. $1(\mathrm{~d})$. As the sets $R_{D}$ and $S_{D}$ are also empty, suppose that all vertices $t_{i}$ are placed in the region of $D\left(W_{5}\right)$ with four vertices $v_{1}, v_{2}, v_{3}$, and $v_{6}$ of $W_{5}$ on its boundary according to a certain symmetry of this drawing $W_{5}$. Consequently, we can apply the same idea as in the previous subcase regarding the existence or non existence of a subgraph $T^{i} \in T_{D}$ which does not cross the edges of $C_{5}^{\star}$.

In addition, without loss of generality, we can consider the drawing of $W_{5}$ with the vertex notation in such a way as shown in Fig. 1(e). Clearly, all vertices $t_{i}$ are placed in the region of $D\left(W_{5}\right)$ with five vertices $v_{1}, v_{2}, v_{3}, v_{5}$, and $v_{6}$ of $W_{5}$ on its boundary, and the set $R_{D}$ is empty but the set $S_{D}$ can be nonempty. So, two possible subcases may occur:

a) Let $S_{D}$ be the nonempty set, that is, only the edge $v_{1} v_{2}$ of $W_{5}$ is crossed by the edge $t_{i} v_{4}$ of each subgraph $T^{i} \in S_{D}$. It is not difficult to verify in six possible regions of $D\left(W_{5} \cup T^{i}\right)$ that $\operatorname{cr}_{D}\left(W_{5} \cup T^{i}, T^{k}\right) \geq 6$ holds for any subgraph $T^{k}, k \neq i$. By fixing the subgraph $W_{5} \cup T^{i}$, we obtain a contradiction with the assumption (3.3) in $D$.

b) Let $S_{D}$ be the empty set, that is, each subgraph $T^{i}$ crosses the edges of $W_{5}$ at least twice. The edges of $C_{5}^{\star}$ are not crossed by any $T^{i} \in T_{D}$ only if either $\operatorname{rot}_{D}\left(t_{i}\right)=$ $(123465)$ or $\operatorname{rot}_{D}\left(t_{i}\right)=(123645)$. In both cases, $\operatorname{cr}_{D}\left(W_{5} \cup T^{i}, T^{k}\right) \geq 6$ is fulfilling for any subgraph $T^{k}, k \neq i$, which yields a contradiction by fixing the subgraph $W_{5} \cup T^{i}$. If there is no subgraph $T^{i} \in T_{D}$ with $\operatorname{cr}_{D}\left(C_{5}^{\star}, T^{i}\right)=0$, then the discussed drawing contradicts the assumption of $D$ again by Lemma 3.2.

Finally, without loss of generality, we assume the drawing of $W_{5}$ with the vertex notation in such a way as shown in Fig. 1(f). The set $R_{D}$ is empty, but the set $S_{D}$ can be nonempty, and so the proof proceeds in a similar way as for the drawing of $W_{5}$ in Fig. 1(e). Only if $S_{D} \neq \emptyset$, there are two possibilities of obtaining a subgraph $T^{i} \in S_{D}$ with either $\operatorname{rot}_{D}\left(t_{i}\right)=(123645)$ or $\operatorname{rot}_{D}\left(t_{i}\right)=(123654)$.

Case 4: $\mathrm{cr}_{D}\left(W_{5}\right)=3$. If we consider the drawing of $W_{5}$ as in Fig. $1(\mathrm{~g})$, by applying the same process as for the drawing in Fig. 1(b), we obtain at least $\left\lceil\frac{n}{2}\right\rceil+1$ subgraphs $T^{i} \in T_{D}$ whose edges cross the edges of $C_{5}^{\star}$. Otherwise, if we assume the drawing of $W_{5}$ as shown in Fig. 1(h), then the same idea as in such a case in [3] regarding the existence or non existence of a subgraph $T^{i} \in R_{D}$ could be exploited.

We have shown, in all cases, that there are at least $6\left\lfloor\frac{n}{2}\right\rfloor\left\lfloor\frac{n-1}{2}\right\rfloor+n+3\left\lfloor\frac{n}{2}\right\rfloor+1$ crossings in each good drawing $D$ of the graph $W_{5}+P_{n}$. This completes the proof of Theorem 3.3.

\section{CONCLUSIONS}

Staš and Valiska were able to postulate that

$$
\operatorname{cr}\left(W_{m}+P_{n}\right)=Z(m+1) Z(n)+(Z(m)-1)\left\lfloor\frac{n}{2}\right\rfloor+n+1 \quad \text { for all } m \geq 3, n \geq 2,
$$

where $Z(n)=\left\lfloor\frac{n}{2}\right\rfloor\left\lfloor\frac{n-1}{2}\right\rfloor$ is Zarankiewicz's number. Recently, this conjecture was proved for the graph $W_{3}+P_{n}$ by Klešč and Schrötter [17], and for the graph $W_{4}+P_{n}$ by Staš and Valiska [25]. Theorem 3.3 also confirms the validity of this conjecture for $W_{5}+P_{n}$.

Theorem 4.4 ([17], Theorem 4.2). $\operatorname{cr}\left(W_{3}+P_{n}\right)=2\left\lfloor\frac{n}{2}\right\rfloor\left\lfloor\frac{n-1}{2}\right\rfloor+n+1$ for $n \geq 2$.

Theorem 4.5 ([25], Theorem 3.3). $\operatorname{cr}\left(W_{4}+P_{n}\right)=4\left\lfloor\frac{n}{2}\right\rfloor\left\lfloor\frac{n-1}{2}\right\rfloor+n+\left\lfloor\frac{n}{2}\right\rfloor+1$ for $n \geq 2$.

On the other hand, the graphs $W_{m}+P_{2}$ and $W_{m}+P_{3}$ are isomorphic to the join product of the cycle $C_{m}$ with the cycle $C_{3}$ and with the graph $K_{4} \backslash e$ obtained by removing one edge from the complete graph $K_{4}$, respectively. The exact values for the crossing numbers of the graphs $C_{m}+C_{n}$ are given by Klešč [11], that is, $\operatorname{cr}\left(C_{m}+C_{n}\right)=Z(m) Z(n)+2$ 
for any $m, n \geq 3$ with $\min \{m, n\} \leq 6$. The crossing numbers of $K_{4} \backslash e+C_{m}$ equal to $2\left\lfloor\frac{m}{2}\right\rfloor\left\lfloor\frac{m-1}{2}\right\rfloor+\left\lfloor\frac{m}{2}\right\rfloor+3$ were established also by Klešč [12]. Further, the graph $W_{m}+P_{4}$ is isomorphic to the join product of the cycle $C_{m}$ with the graph $K_{1,4}+3 e$ obtained by adding three non incident edges with the same vertex to the complete bipartite graph $K_{1,4}$. Using the result of Staš [23], the crossing numbers of the graphs $\left(K_{1,4}+3 e\right)+C_{m}$ are given by $4\left\lfloor\frac{m}{2}\right\rfloor\left\lfloor\frac{m-1}{2}\right\rfloor+2\left\lfloor\frac{m}{2}\right\rfloor+3$ for each $m \geq 3$. These facts allow us to determine another results for the join product of the wheels $W_{m}$ with the path on two, three, and four vertices.

Theorem 4.6 ([25], Theorem 5.1). $\operatorname{cr}\left(W_{m}+P_{2}\right)=\left\lfloor\frac{m}{2}\right\rfloor\left\lfloor\frac{m-1}{2}\right\rfloor+2$ for $m \geq 3$.

Theorem 4.7 ([25], Theorem 5.2). $\operatorname{cr}\left(W_{m}+P_{3}\right)=2\left\lfloor\frac{m}{2}\right\rfloor\left\lfloor\frac{m-1}{2}\right\rfloor+\left\lfloor\frac{m}{2}\right\rfloor+3$ for $m \geq 3$.

Theorem 4.8 ([23], Corollary 7). $\operatorname{cr}\left(W_{m}+P_{4}\right)=4\left\lfloor\frac{m}{2}\right\rfloor\left\lfloor\frac{m-1}{2}\right\rfloor+2\left\lfloor\frac{m}{2}\right\rfloor+3$ for $m \geq 3$.

One can easily verify that these results also confirm the validity of this conjecture for the graphs $W_{m}+P_{2}, W_{m}+P_{3}$, and $W_{m}+P_{4}$.

Acknowledgment. This work was supported by the Slovak Research and Development Agency under the contract No. APVV-18-0373 and by the Scientific Grant Agency (VEGA) under contract No. 1/0584/20.

\section{REFERENCES}

[1] Berežný, Š.; Staš, M. Cyclic permutations and crossing numbers of join products of symmetric graph of order six. Carpathian J. Math. 34 (2018), no. 2, 143-155.

[2] Berežny, Š.; Staš, M. Cyclic permutations and crossing numbers of join products of two symmetric graphs of order six. Carpathian J. Math. 35 (2019), no. 2, 137-146.

[3] Berežný, Š.; Staš, M. On the crossing number of join of the wheel on six vertices with the discrete graph. Carpathian J. Math. 36 (2020), no. 3, 381-390.

[4] Clancy, K.; Haythorpe, M.; Newcombe, A. A survey of graphs with known or bounded crossing numbers. Australasian J. Combin. 78 (2020), no. 2, 209-296.

[5] Draženská, E. On the crossing number of join of graph of order six with path, Proc. CJS 2019: $22^{\text {th }}$ Czech-Japan Seminar on Data Analysis and Decision Making, (2019), 41-48.

[6] Draženská, E. Crossing numbers of join product of several graphs on 6 vertices with path using cyclic permutation. In Proceedings of the MME 2019: Proceedings of the $37^{\text {th }}$ international conference, České Budějovice, Czechia, 11-13 September 2019; pp. 457-463.

[7] Garey, M. R.; Johnson, D. S. Crossing number is NP-complete. SIAM J. Algebraic. Discrete Methods 4 (1983), no. 3, 312-316.

[8] Hernández-Vélez, C.; Medina, C.; Salazar, G. The optimal drawing of $K_{5, n}$. Electron. J. Combin. 21 (2014), no. $4, \sharp P 4.1,29$ pp.

[9] Kleitman, D. J. The crossing number of $K_{5, n}$. J. Combinatorial Theory 9 (1970), 315-323.

[10] Klešč, M. The crossing numbers of join of the special graph on six vertices with path and cycle. Discrete Math. 310 (2010), no. 9, 1475-1481.

[11] Klešč, M. The join of graphs and crossing numbers. Electron. Notes in Discrete Math. 28 (2007), 349-355.

[12] Klešč, M. The crossing numbers of join of cycles with graphs of order four. Proc. Aplimat 2019: $18^{\text {th }}$ Conference on Applied Mathematics, (2019), 634-641.

[13] Klešč, M.; Kravecová, D.; Petrillová, J. The crossing numbers of join of special graphs. Electrical Engineering and Informatics 2: Proceeding of the Faculty of Electrical Engineering and Informatics of the Technical University of Košice, (2011), 522-527.

[14] Klešč, M.; Kravecová, D.; Petrillová, J. On the crossing numbers of Cartesian products of paths with special graphs. Carpathian J. Math. 30 (2014), no. 3, 317-325.

[15] Klešč, M.; Petrillová, J.; Valo, M. Minimal number of crossings in strong product of paths. Carpathian J. Math. 29 (2013), no. 1, 27-32.

[16] Klešč, M.; Schrötter, $\breve{S}$. The crossing numbers of join of paths and cycles with two graphs of order five. Combinatorial Algorithms Springer, LNCS, 7125 (2012), 160-167.

[17] Klešč, M.; Schrötter, Š. The crossing numbers of join products of paths with graphs of order four. Discuss. Math. Graph Theory 31 (2011), no. 2, 321-331. 
[18] Klešč, M.; Staš, M. Cyclic permutations in determining crossing numbers. Discuss. Math. Graph Theory [to appear]

[19] Mei, H.; Huang, Y. The Crossing Number of $K_{1,5, n}$. Int. J. Math. Combin. 1 (2007), no. 1, $33-44$.

[20] Ouyang, Z.; Wang, J.; Huang, Y. The crossing number of join of the generalized Petersen graph $P(3,1)$ with path and cycle. Discuss. Math. Graph Theory 38 (2018), no. 2, 351-370.

[21] Su, Z.; Huang Y. The Crossing Number of $W_{m} \vee P_{n}$. J. Math. Stud. 45 (2012), no. 3, 310-314.

[22] Staš, M. Determining crossing number of join of the discrete graph with two symmetric graphs of order five. Symmetry 11 (2019), no. 2, 123.

[23] Staš, M. The crossing numbers of join products of paths and cycles with four graphs of order five. Mathematics 9 (2021), no. 11, 1277.

[24] Staš, M.; Petrillová, J. On the join products of two special graphs on five vertices with the path and the cycle. J. Math. Model. and Geometry 6 (2018), no. 2, 1-11.

[25] Staš, M.; Valiska, J. On the crossing numbers of join products of $W_{4}+P_{n}$ and $W_{4}+C_{n}$. Opuscula Math. 41 (2021), no. 1, 95-112.

[26] Woodall, D. R. Cyclic-order graphs and Zarankiewicz's crossing number conjecture. J. Graph Theory 17 (1993), no. 6, 657-671.

\author{
DEPARTMENT OF MATHEMATICS AND THEORETICAL INFORMATICS \\ FACULTY OF ELECTRICAL ENGINEERING AND INFORMATICS \\ TECHNICAL UNIVERSITY OF KOŠICE \\ KOŠICE, SLOVAKIA \\ Email address: stefan.berezny@tuke.sk \\ Email address: michal.stas@tuke.sk
}

